\title{
Orthogonal Design Method for Optimizing Roughly Designed Antenna
}

\author{
Qing Zhang, ${ }^{1}$ Sanyou Zeng, ${ }^{2}$ and Chunbang $\mathrm{Wu}^{3}$ \\ ${ }^{1}$ School of Mathematics \& Computer Science, Huanggang Normal University, Huanggang, Hubei 38000, China \\ ${ }^{2}$ School of Computer Science, China University of GeoSciences, Wuhan, Hubei 430074, China \\ ${ }^{3}$ China Academy of Space Technology (Xián), Xián, Shanxi 710000, China
}

Correspondence should be addressed to Sanyou Zeng; sanyouzeng@gmail.com

Received 12 January 2014; Accepted 5 February 2014; Published 17 March 2014

Academic Editor: Dau-Chyrh Chang

Copyright (C) 2014 Qing Zhang et al. This is an open access article distributed under the Creative Commons Attribution License, which permits unrestricted use, distribution, and reproduction in any medium, provided the original work is properly cited.

\begin{abstract}
Orthogonal design method (ODM) is widely used in real world application while it is not used for antenna design yet. It is employed to optimize roughly designed antenna in this paper. The geometrical factors of the antenna are relaxed within specific region and each factor is divided into some levels, and the performance of the antenna is constructed as objective. Then the ODM samples small number of antennas over the relaxed space and finds a prospective antenna. In an experiment of designing ST5 satellite miniantenna, we first get a roughly evolved antenna. The reason why we evolve roughly is because the evolving is time consuming even if numerical electromagnetics code 2 (NEC2) is employed (NEC2 source code is openly available and is fast in wire antenna simulation but not much feasible). Then the ODM method is employed to locally optimize the antenna with HFSS (HFSS is a commercial and feasible electromagnetics simulation software). The result shows the ODM optimizes successfully the roughly evolved antenna.
\end{abstract}

\section{Introduction}

Orthogonal design method (ODM) has been widely researched. Literature $[1,2]$ surveys this method in both theoretical and applied way. The ODM samples a small number of evenly distributed points over a large search space. Then it statistically summarizes a prospective good solution. The application of this method is much wide, such as in, for example, chemical and biological fields $[3,4]$, image process [5], laser polishing [6], software testing technique [7], algorithm [8], semiconductor manufacturing [9], optics [10], and robust design [11]. Recent theory research on the ODM method can be found still, for example, the mixed-level orthogonal array research in [12].

The current practice of designing antennas by hand is limited in its ability to develop new and better antenna designs because it requires significant domain expertise and experience and is both time and labor intensive. With this approach, an antenna engineer will select a particular class of antennas and then spend weeks or months testing and adjusting a design, mostly in simulation using electromagnetic modeling software. As an alternative, researchers have been investigating evolutionary antenna optimization since the early 1990s. For example, genetic algorithm/evolutionary algorithm ([13]) is adopted to optimize antenna [14-16], particle swarm optimization ([17-19]) to optimize antenna $[20,21]$, and differential evolution $([22,23])$ to optimize antenna [24].

A run of evolutionary algorithm in designing an antenna usually takes over 10,000 electromagnetic simulations while a simulation usually takes minutes or even hours. Then fast computing and incomplete simulation are adopted to reduce running time. The output of such evolution can only be called a roughly evolved antenna.

This paper focuses on optimizing locally this kind of rough antennas. We first relax the geometrical factors of the rough antenna within specific regions, divide each factor into some levels, and construct objective by using the performance of the antenna. Then we sample small number of antennas over the relaxed space and find a prospective antenna. It is the method that we call orthogonal design method (ODM).

In an experiment of designing ST5 satellite antenna, we first get a roughly evolved antenna with NEC2. Then the 
ODM is employed to locally optimize the antenna with HFSS. The result shows the ODM method optimizes successfully the roughly evolved antenna.

The remainder of this paper is organized as follows. Section 2 introduces the principal of the ODM method by using an example. Section 3 presents the fact that the ODM method optimizes antenna locally. Designing NASA ST5 antenna is used to test the ODM method in Section 4. The paper is concluded in Section 5.

\section{Principal of Orthogonal Design Method}

2.1. An Example to Introduce Orthogonal Design Method. We use a concrete example in this subsection to introduce the basic concept of an "orthogonal design method". For further details, see [25]. The example is concerned with the yield of vegetable growth. The yield of a vegetable depends on at least three factors:

(1) the temperature,

(2) the amount of fertilizer used,

(3) the $\mathrm{pH}$ value of the soil.

In this example, each factor has three possible values, as shown in Table 1. We say that each factor has three "levels."

The objective is to find the best combination of levels for a maximum yield. We can perform an experiment for each combination and then select the combination with the highest yield. In the above example, there are $3 \times 3 \times$ $3=27$ combinations, and hence there are 27 experiments. In general, when there are $N$ factors, each with $Q$ levels, there are $Q^{N}$ possible combinations. When $N$ and $Q$ are large, it may not be possible to perform all $Q^{N}$ experiments. Therefore, it is desirable to sample a small, but representative, set of combinations, for the experimentation. The "orthogonal design method" was developed for this purpose [25], where an orthogonal array is constructed to represent the sampled set of combinations which evenly distribute over the experimentation space. An orthogonal array $L_{M}\left(Q^{N}\right)$ is a $M \times N$ array with $Q$ levels for each column, denoted by $\{1,2, \ldots, Q\}$. We select $M$ combinations to be tested, where $M$ may be much smaller than $Q^{N}$. Equation (1) is an example of an orthogonal array where $M=9, N=3$, and $Q=3$. Figure 1 shows the 9 representative combinations (marked with " $\Delta$ ") evenly distributed over all the 27 combinations. Consider

$$
L_{9}\left(3^{3}\right)=\left[\begin{array}{lll}
1 & 1 & 1 \\
1 & 2 & 2 \\
1 & 3 & 3 \\
2 & 1 & 2 \\
2 & 2 & 3 \\
2 & 3 & 1 \\
3 & 1 & 3 \\
3 & 2 & 1 \\
3 & 3 & 2
\end{array}\right]
$$

The $L_{9}\left(3^{3}\right)$ has three factors, three levels per factor, and nine combinations of levels. The three factors have respective
TABLE 1: Experimental design with three factors and three levels per factor.

\begin{tabular}{lccc}
\hline Levels & \multicolumn{3}{c}{ Factor } \\
& Temperature $\left({ }^{\circ} \mathrm{C}\right)$ & Fertilizer $\left(\mathrm{g} / \mathrm{m}^{2}\right)$ & $\mathrm{pH}$ \\
\hline Level 1 & 20 & 100 & 6 \\
Level 2 & 25 & 150 & 7 \\
Level 3 & 30 & 200 & 8 \\
\hline
\end{tabular}

$\backslash$ Construct the basic columns as follows.

FOR $k=1$ to $J$

$$
\begin{aligned}
& j=\left(Q^{k-1}-1\right) /(Q-1)+1 \\
& \text { FOR } i=1 \text { to } Q^{J} \\
& a_{i, j}=\text { floor }\left((i-1) /\left(Q^{J-k}\right)\right) \bmod Q \\
& \text { ENDFOR }
\end{aligned}
$$

ENDFOR

$\backslash$ Construct the non-basic columns as follows.

FOR $k=2$ to $J$

$$
\begin{aligned}
& j=\left(Q^{k-1}-1\right) /(Q-1)+1 ; \\
& \text { FOR } s=1 \text { to } j-1, t=1 \text { to } Q-1 \\
& \quad \vec{a}_{j+(s-1)(Q-1)+t}=\left(\vec{a}_{s} \times t+\vec{a}_{j}\right) \bmod Q ; \\
& \text { ENDFOR }
\end{aligned}
$$

ENDFOR

Algorithm 1: Constructing the orthogonal array $L_{M}\left(Q^{P}\right)$.

levels 1,1 , and 1 in the first combination, 1,2 , and 2 in the second combination, and so forth. We apply the orthogonal array $L_{9}\left(3^{3}\right)$ to select nine combinations to be tested. The nine combinations and their yields in the above example are shown in Table 2.

From the yields of the selected combinations, a promising solution can be obtained by the following statistical method.

(1) Calculate the mean value of the yields for each factor at each level, where each factor has a level with the best mean value (cf. Algorithm 2).

The mean yields of the temperature are

$\Gamma_{1,1}=(2.75+4.52+4.65) / 3=3.97$ at level $1\left(20^{\circ} \mathrm{C}\right)$,

$\Gamma_{2,1}=(4.60+5.58+4.10) / 3=4.76$ at level $2\left(25^{\circ} \mathrm{C}\right)$,

$\Gamma_{3,1}=(5.32+4.10+4.37) / 3=4.60$ at level $3\left(30^{\circ} \mathrm{C}\right)$.

The mean yields of the fertilizer are

$\Gamma_{1,2}=(2.75+4.60+5.32) / 3=4.22$ at level $1\left(100 \mathrm{~g} / \mathrm{m}^{2}\right)$,

$\Gamma_{2,2}=(4.52+5.58+4.10) / 3=4.73$ at level $2\left(150 \mathrm{~g} / \mathrm{m}^{2}\right)$,

$\Gamma_{3,2}=(4.65+4.10+4.37) / 3=4.37$ at level $3\left(200 \mathrm{~g} / \mathrm{m}^{2}\right)$.

The mean yields of the $\mathrm{PH}$ value are

$\Gamma_{1,3}=(2.75+4.10+4.10) / 3=3.65$ at level $1(6)$,

$\Gamma_{2,3}=(4.52+4.60+4.37) / 3=4.50$ at level $2(7)$,

$\Gamma_{3,3}=(4.65+5.58+5.32) / 3=5.18$ at level 3(8).

These mean yields are shown in Table 3 . 
TABLE 2: The yield of nine representative combinations, based on the orthogonal array $L_{9}\left(3^{3}\right)$.

\begin{tabular}{lcccc}
\hline No. & & Factor & $\mathrm{pH}$ & $1(6)$ \\
\hline 1 & Temperature & Fertilizer & $2(7)$ & \\
2 & $1\left(20^{\circ} \mathrm{C}\right)$ & $1\left(100 \mathrm{~g} / \mathrm{m}^{2}\right)$ & $3(8)$ & \\
3 & $1\left(20^{\circ} \mathrm{C}\right)$ & $2\left(150 \mathrm{~g} / \mathrm{m}^{2}\right)$ & $2(7)$ & 4.52 \\
4 & $1\left(20^{\circ} \mathrm{C}\right)$ & $3\left(200 \mathrm{~g} / \mathrm{m}^{2}\right)$ & $3(8)$ & 4.65 \\
5 & $2\left(25^{\circ} \mathrm{C}\right)$ & $1\left(100 \mathrm{~g} / \mathrm{m}^{2}\right)$ & $1(6)$ & 5.60 \\
6 & $2\left(25^{\circ} \mathrm{C}\right)$ & $2\left(150 \mathrm{~g} / \mathrm{m}^{2}\right)$ & $3(8)$ & 4.10 \\
7 & $2\left(25^{\circ} \mathrm{C}\right)$ & $3\left(200 \mathrm{~g} / \mathrm{m}^{2}\right)$ & $1(6)$ & 4.32 \\
8 & $3\left(30^{\circ} \mathrm{C}\right)$ & $1\left(100 \mathrm{~g} / \mathrm{m}^{2}\right)$ & $2(7)$ & 4.37 \\
9 & $3\left(30^{\circ} \mathrm{C}\right)$ & $2\left(150 \mathrm{~g} / \mathrm{m}^{2}\right)$ & & \\
\hline
\end{tabular}

TABLE 3: The mean yield for each factor at different levels.

\begin{tabular}{lccc}
\hline Levels & Temperature & $\begin{array}{c}\text { Mean yield } \\
\text { Fertilizer }\end{array}$ & $\mathrm{pH}$ \\
\hline Level 1 & 3.97 & 4.22 & 3.65 \\
Level 2 & 4.76 & 4.73 & 4.50 \\
Level 3 & 4.60 & 4.37 & 5.18 \\
\hline
\end{tabular}

$\backslash \backslash$ Sum the objective results for each factor at each level

set $\Gamma_{i, j}=0$ for $i=1,2, \ldots, Q ; j=1,2, \ldots, N$

FOR $i=1$ to $M, j=1$ to $N$

$q=a_{i, j} ; \Gamma_{q, j}=\Gamma_{q, j}+y_{i}$

ENDFOR

$\backslash$ Average the results for each factor at each level

$\left[\Gamma_{k, j}\right]_{\mathrm{Q} \times N}=\left[\Gamma_{k, j}\right]_{\mathrm{Q} \times N} \times \mathrm{Q} / M$

Algorithm 2: Calculation of mean value $\left[\Gamma_{k, j}\right]_{\mathrm{Q} \times N}$.

(2) Choose the combination of the best levels as a promising solution (cf. Algorithm 3).

The temperature has the best mean yield, 4.76 , at level 2 (i.e., $25^{\circ} \mathrm{C}$ ). The fertilizer has the best yield, 4.73 , at level 2 (i.e., $150 \mathrm{~g} / \mathrm{m}^{2}$ ). The $\mathrm{pH}$ value has the best yield, 5.18 , at level 3 (i.e., 8). We therefore consider $\left(25^{\circ} \mathrm{C}\right.$, $\left.150 \mathrm{~g} / \mathrm{m}^{2}, 8\right)$ to be a promising and robust solution. The solution may not be optimal when used with an orthogonal design. But for additive and quadratic models, it is provably optimal.

\subsection{A Definition of Orthogonal Array}

Definition 1 (an orthogonal array). $L_{M}\left(Q^{N}\right)$ is a $M \times N$ array with $Q$ levels for each column, denoted by $\{1,2, \ldots, Q\}$. Denote the orthogonal array $L_{M}\left(Q^{N}\right)$ by $\left[a_{i, j}\right]_{M \times N}$ as follows.

(1) In any column $\left\{a_{1, j}, a_{2, j}, \ldots, a_{M, j}\right\}$, each of the $Q$ symbols $1,2, \ldots, Q$ occurs the same number of times; that is, $(M / Q), j=1,2, \ldots, N$.

(2) In any two different columns $\left\{\left(a_{1, j}, a_{1, k}\right),\left(a_{2, j}, a_{2, k}\right)\right.$, $\left.\ldots,\left(a_{M, j}, a_{M, k}\right)\right\}$, each of the $Q^{2}$ possible pairs

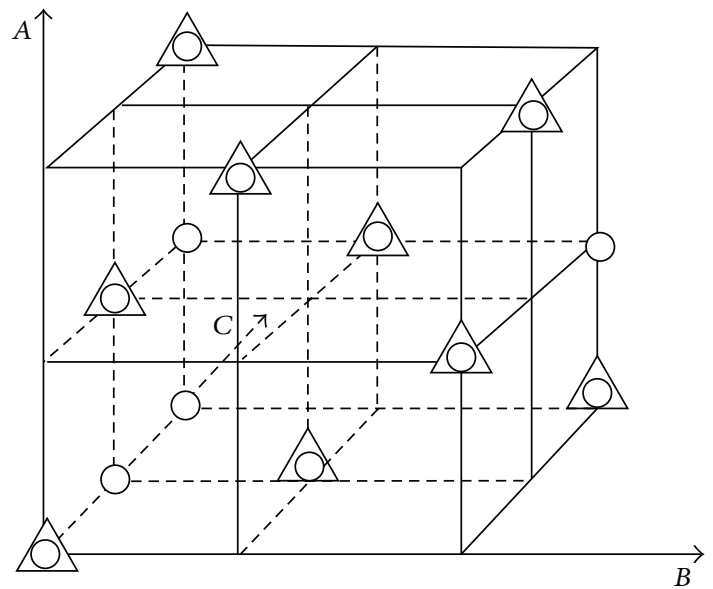

FIGURE 1: Schematic diagram of orthogonal experimental design.

$$
\begin{aligned}
& \text { FOR } j=1 \text { to } N \\
& \quad b_{j}=\arg \max _{i \in\{1,2, \ldots, Q\}} \Gamma_{i, j}
\end{aligned}
$$

ENDFOR

RETURN prospective good combination $\vec{b}=\left(b_{1}, b_{2}, \ldots, b_{N}\right)$

Algorithm 3: Calculation of prospective good combination $\left[b_{j}\right]_{1 \times N}$.

$\{(1,1),(1,2), \ldots,(1, Q),(2,1),(2,2), \ldots,(2, Q), \ldots,(Q$, $1),(Q, 2), \ldots,(Q, Q)\}$ occurs the same number of times $\left(M / Q^{2}\right), j, k=1, \ldots, N, j \neq k$.

Every row of $L_{M}\left(Q^{N}\right)=\left[a_{i, j}\right]_{M \times N}$ represents a different combination of levels, where $a_{i, j}$ means that the $j$ th factor in the $i$ th combination has a level value $a_{i, j}$, and $a_{i, j}$ takes a value from the set $\{1,2, \ldots, Q\}$.

\section{Orthogonal Design Method Designing Antenna}

3.1. Antenna Design Using Orthogonal Design Method. There are many antenna classes, such as reflector antennas (e.g., dish antennas), phased array antennas (consisting of multiple 


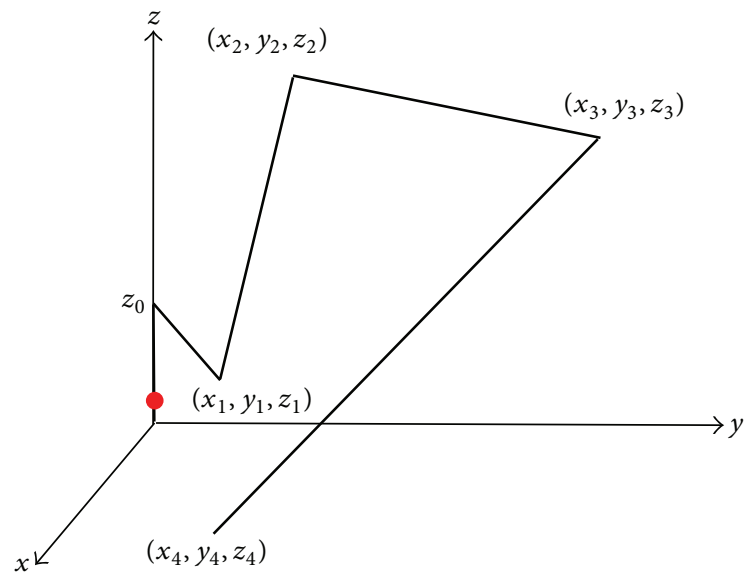

FIGURE 2: Geometric structure of the antenna in the first quadrant.

regularly spaced elements), wire antennas, horn antennas, and microstrip and patch antennas. Each of these classes uses different structures and exploits different properties of electromagnetic waves.

Using orthogonal design method to design antenna, three components are required to be determined: factors, levels, and optimization objective.

The geometrical structure of an antenna is usually regarded as factors. For example, a $N$ element Yagi antenna has $N$ element lengths $\left(l_{1}, l_{2}, \ldots, l_{N}\right), N-1$ spacing distances between elements $\left(d_{1}, d_{2}, \ldots, d_{N-1}\right)$, and one wire radius $(r)$, giving $2 N$ factors total; a conventional rectangular microstrip patch antenna usually has 6 factors: patch length $(l)$ and width $(w)$, substrate height $(h)$ and its dielectric constant $\left(\varepsilon_{r}\right)$, and probe point $(\Delta l, \Delta w)$, the distance from the left-bottom corner of the rectangular microstrip.

Determination of levels for each factor depends on the design specification of the antenna and empirical design.

The optimization objective is to find an antenna best matching the specification (gain, VSWR, etc.). It is a function of the factors. The objective value of an antenna may be achieved by measuring the prototype which is expensive or by simulating the antenna by using electromagnetic simulation softwares The latter one is usually adopted to avoid expensive cost.

Then the antenna design using orthogonal design method is an optimization problem; the formulation of optimizing antenna is defined in the following.

Definition 2 (formulation of optimizing antenna using orthogonal design method). Suppose there are $N$ antenna design factors $x_{1}, x_{2}, \ldots, x_{N}$ and each factor $x_{i}$ has $Q$ levels $x_{i, 1}, x_{i, 2}, \ldots, x_{i, \mathrm{Q}}, i=1,2, \ldots, N$, giving $N^{\mathrm{Q}}$ combinations total. Each combination determines an antenna; there are $N^{Q}$ antennas in all, which is called search space denoted as $\Omega$. The performance of a combination (an antenna) is evaluated by the value of objective $f(\vec{x})$. The bigger the value of $f(\vec{x})$ the better the performance of the antenna in maximization formulation, $\vec{x} \in \Omega$. In this way, the optimization is Max $f(\vec{x})$ where $\vec{x} \in \Omega$
Note. Q must be prime in this paper; see Section 3.2.

3.2. Creating Orthogonal Array. As we will explain shortly, the technique proposed in this paper usually requires different orthogonal arrays for different problems. The construction of orthogonal array is not a trivial task since we do not know whether an orthogonal array of given size exists. Many orthogonal arrays have been presented in the literatures. It is impossible, however, to tabulate them all. For the necessity of the technique in this paper, we introduce a simple permutation method that is derived from the mathematical theory of Galois fields (see [1]), to construct a class of orthogonal arrays $L_{M}\left(Q^{P}\right)$. The $M, P, Q$ fulfill the following:

$$
\begin{gathered}
M=Q^{J}, \\
P=\frac{\left(Q^{J}-1\right)}{(Q-1)},
\end{gathered}
$$

where $Q$ is prime and $J$ is a positive integer.

Denote the $j$ th column of the orthogonal array $\left[a_{i, j}\right]_{M \times P}$ by $\vec{a}_{j}$. Columns $\vec{a}_{j}$ for $j=1,2,\left(Q^{2}-1\right) /(Q-1)+1,\left(Q^{3}-\right.$ $1) /(Q-1)+1, \ldots,\left(Q^{J-1}-1\right) /(Q-1)+1$ are called the basic columns. The others are called the nonbasic columns. The algorithm first constructs the basic columns and then generates the nonbasic columns. The details are given in the Algorithm 1.

3.3. Determining the Size of the Needed Orthogonal Array. The $L_{M}\left(Q^{P}\right)$ constructed by Algorithm 1 has a size of $M$ combinations (antennas). It can be adopted for a problem with $Q$ levels and $N$ factors where $N \leq P$. The $M$ and $P$ in $L_{M}\left(Q^{P}\right)$ are determined by given $Q$ and $J$ according to (2), while in an antenna design problem the number of level $Q$ and the number of factor $N$ are given according to Definition 2. Then the $J$ is demanded to be determined to construct an orthogonal array for the problem.

$M$ combinations mean $M$ electromagnetic simulations each of which is time consuming. We choose the $M$ as small as possible. This can be done by choosing the $J$ as small as possible according to (2). Then the $J$ is determined by

$$
\begin{aligned}
& \text { Min } \quad J \\
& \text { st. } \quad P=\frac{\left(Q^{I}-1\right)}{(Q-1)} \geq N, \quad J=2,3, \ldots
\end{aligned}
$$

The $L_{M}\left(Q^{P}\right)$, constructed by Algorithm 1, has $P$ columns. For a problem with $N$ factors, we discard the last $P-N$ columns of $L_{M}\left(Q^{P}\right)$ and obtain an array $L_{M}\left(Q^{N}\right)$ which is still orthogonal according to Definition 1.

For the problem of vegetable growth, there are 3 factors $(N=3)$ and 3 levels $(Q=3) . J=2$ by $(3)$ and the orthogonal 
array is $L_{9}\left(3^{4}\right)$ with 4 columns $(P=4)$. We discard the last column of the $L_{9}\left(3^{4}\right)$ and get the needed array $L_{9}\left(3^{3}\right)$ :

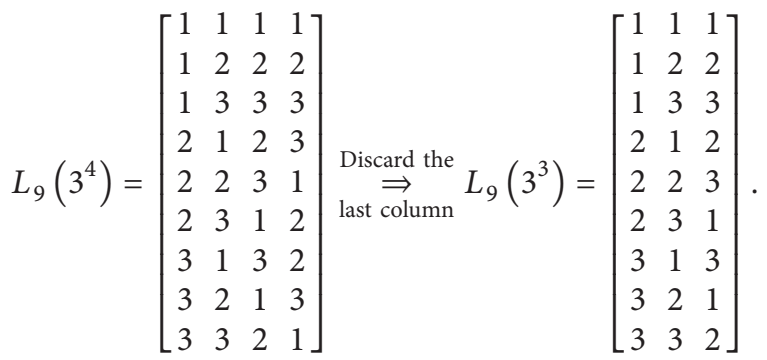

3.4. Doing Orthogonal Experiments. The $M$ combinations (antennas) are evaluated by orthogonal experiments (electromagnetic simulations). We obtain $M$ objective values (performances of the antennas) denoted as $\left[y_{i}\right]_{M \times 1}$ where the objective has the value $y_{i}$ at the $i$ th combination. It is similar to fill out Table 2 for the above vegetable example.

The best combination (antenna) among the $M$ combinations, denoted as $\vec{b}^{*}=\left(b_{1}^{*}, b_{2}^{*}, \ldots, b_{N}^{*}\right)$, is usually not a global optimal solution. A prospective better solution could be found by using statistical method in the following.

3.5. Calculating Mean Objective Value at Each Level of Each Factor. Denote $\Gamma_{k, j}$ as the mean objective value at the $k$ th level of the $j$ th factor; $k=1,2, \ldots, Q, j=1,2, \ldots, N$. Consider

$$
\Gamma_{k, j}=\frac{Q}{M} \sum_{a_{i, j}=k} y_{i}
$$

where the orthogonal array $L_{M}\left(Q^{N}\right)$ has the value $a_{i, j}$ at the $i$ th row and $j$ th column; that is, the $j$ th factor has level $a_{i, j}$ in the $i$ th combination (experiment). The objective has value $y_{i}$ at the $i$ th combination, and $\sum_{a_{i, j}=k} y_{i}$ implies the sum of $y_{i}$ where any $i$ satisfies $a_{i, j}=k$ for given $j$. All those mean values compose a matrix $\left[\Gamma_{k, j}\right]_{Q \times N}$.

For the vegetable example, this calculation will fill out Table 3. The details of the algorithm are shown in Algorithm 2.

3.6. Finding Prospective Good Solution. A best level can be found for each factor from the mean value matrix $\left[\Gamma_{k, j}\right]_{\mathrm{Q} \times N}$. The combination of the best levels is usually guessed better than the best combination $\vec{b}^{*}=\left(b_{1}^{*}, b_{2}^{*}, \ldots, b_{N}^{*}\right)$ among the $M$ simulated combinations. Actually, for additive or quadratic models, it is optimal. The details of calculating the combination of the best levels are given in Algorithm 3.

However, the goodness of the combination $\vec{b}=\left(b_{1}, b_{2}\right.$, $\ldots, b_{N}$ ) is only a guess. We must do experiment (electromagnet simulation) for it to verify its performance. Actually, it is possible that $\vec{b}$ is worse than $\vec{b}^{*}$. In this way, the final output will be the better one between $\vec{b}$ and $\vec{b}^{*}$.
TABLE 4: Antenna design specifications of NASA ST5 antenna.

\begin{tabular}{lc}
\hline Property & Specifications \\
\hline Transmit frequency & $8470 \mathrm{MHz}$ \\
Receive frequency & $7209 \mathrm{MHz}$ \\
Polarization & Right-hand circular \\
Transmit frequency VSWR & $<1.2: 1$ \\
Receive frequency VSWR & $<1.5: 1$ \\
Gain mode & $\geq 0 \mathrm{dBic}, 40^{\circ} \leq \theta \leq 80^{\circ}, 0^{\circ} \leq \varphi \leq 360^{\circ}$ \\
Input impedance & $50 \Omega$ \\
Diameter & $<15.24 \mathrm{~cm}$ \\
Height & $<15.24 \mathrm{~cm}$ \\
Quality & $<165 \mathrm{~g}$ \\
Ground plane diameter & $15.24 \mathrm{~cm}$ \\
\hline
\end{tabular}

\section{Testing ODM by ST5 Antenna Design}

NASA ST5 mission consists of three microsatellites successfully launched in 2006 [26]. The specification of their antennas is shown in Table 4. Antenna designed by evolutionary algorithm was very small and was successfully applied for this mission [27], which is the first application of evolutionary antenna in the region of space science.

In this paper, we first roughly evolved an antenna by using NEC2 to simulate since NEC2 computes fast in simulating wire antennas and its code is openly available. The roughly evolved antenna is shown in left plot of Figure 3. Its gain at frequency $7209 \mathrm{MHz}$ is shown in the left plot of Figure 4, and the one at frequency $8740 \mathrm{MHz}$ is in the left plot of Figure 5 . Its VSWRs are shown in the left column of Table 7. The gains satisfy the specification, but the VSWRs do not.

NEC2 is not much feasible while HFSS is feasible and commercial. Then HFSS software is adopted for ODM to optimize the roughly evolved antenna locally.

4.1. Factors, Levels, and Objective. The antenna is generated by starting with an initial feeder and adding four identical arms. Antenna geometric structure is symmetrical about the $z$-axis and each arm rotated $90^{\circ}$ from its neighbors. We only encode the arm in the first quadrant where $x>0, y>0$, and $z>0$. After constructing the arm in the first quadrant, it is copied three times and these copies are placed in each of the other quadrants through rotations of $90^{\circ} / 180^{\circ} / 270^{\circ}$. Linking such four aims to antenna feeder, we get the complete antenna.

As shown in Figure 2, the arm including the feeder in the first quadrant is 5 segments of conductors linked head-tail. The geometric structure can be coded as follows: the initial feed wire is a thumbnail lead, starting by origin along the positive $z$-axis with end point $\left(0,0, z_{0}\right)$. The other four ends of the wires are $\left(x_{1}, y_{1}, z_{1}\right),\left(x_{2}, y_{2}, z_{2}\right),\left(x_{3}, y_{3}, z_{3}\right)$, and $\left(x_{4}, y_{4}, z_{4}\right)$, respectively; see Figure 2. The radius of the wires is specified as $0.5 \mathrm{~mm}$. Then geometric structure of the antenna can be determined by 13 factors: $z_{0}, x_{1}, y_{1}, z_{1}, x_{2}$, $y_{2}, z_{2}, x_{3}, y_{3}, z_{3}, x_{4}, y_{4}$, and $z_{4}$. 

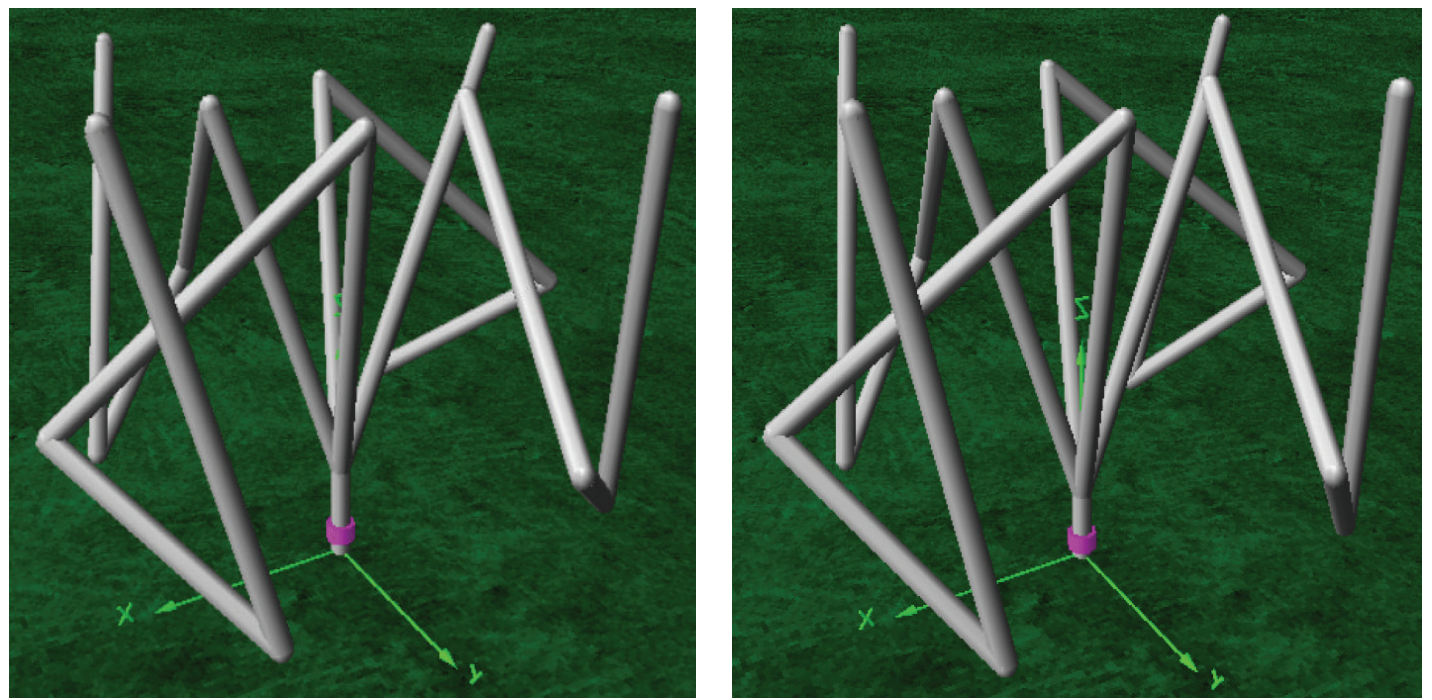

FIGURE 3: Structures of the antenna: the left is the roughly evolved one and the right the orthogonally designed one.

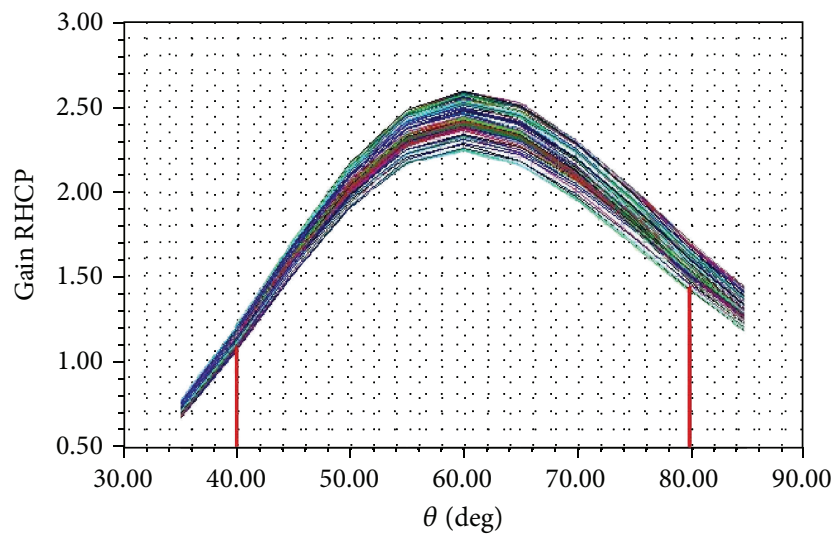

(a)

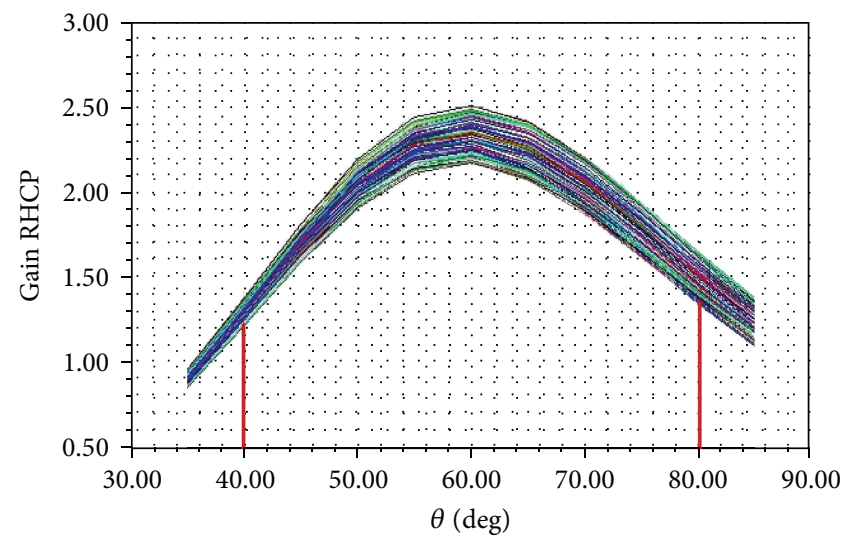

(b)

FIGURE 4: RHCP gain at $7209 \mathrm{MHz}$ frequency: (a) for the roughly evolved antenna and (b) for the orthogonally designed one.

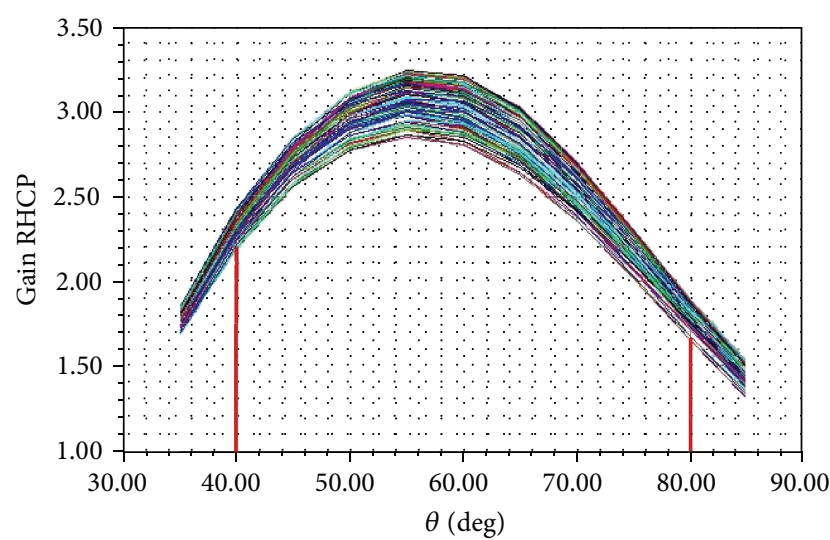

(a)

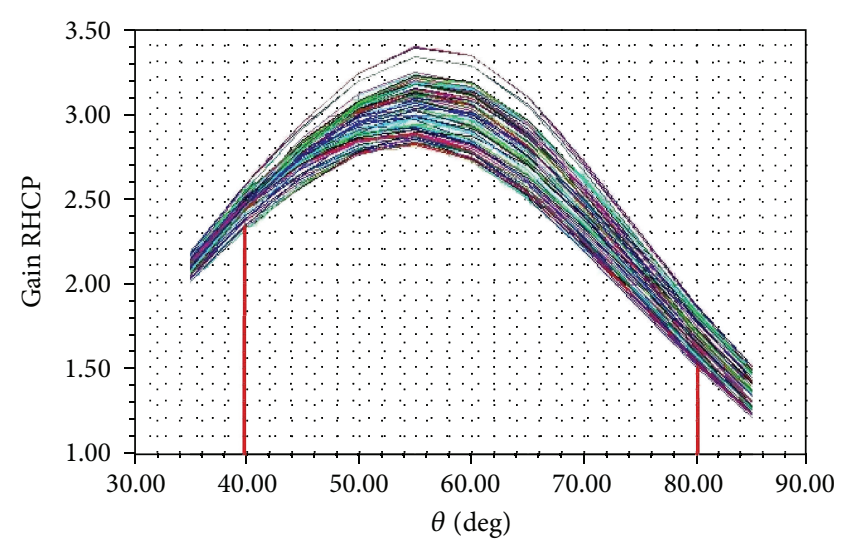

(b)

FIGURE 5: RHCP gain at $8470 \mathrm{MHz}$ frequency: (a) for the roughly evolved antenna and (b) for the orthogonally designed one. 
TABLE 5: ST5 antenna design with 13 factors and 3 levels each.

\begin{tabular}{lccccccccccccc}
\hline Levels & \multicolumn{10}{c}{ Factors } \\
& $1\left(z_{0}\right)$ & $2\left(x_{1}\right)$ & $3\left(y_{1}\right)$ & $4\left(z_{1}\right)$ & $5\left(x_{2}\right)$ & $6\left(y_{2}\right)$ & $7\left(z_{2}\right)$ & $8\left(x_{3}\right)$ & $9\left(y_{3}\right)$ & $10\left(z_{3}\right)$ & $11\left(x_{4}\right)$ & $12\left(y_{4}\right)$ & $13\left(z_{4}\right)$ \\
\hline-1 & 3.00 & 1.50 & 4.87 & 19.13 & 14.02 & 1.50 & 9.04 & 7.72 & 9.20 & 3.07 & 12.47 & 5.91 & 20.50 \\
0 & 3.50 & 2.00 & 5.37 & 19.63 & 14.52 & 2.00 & 9.54 & 8.21 & 9.70 & 3.57 & 12.97 & 6.41 & 20.90 \\
1 & 4.00 & 2.50 & 5.87 & 20.13 & 15.02 & 2.50 & 10.04 & 8.72 & 10.20 & 4.07 & 13.47 & 6.91 & 21.50 \\
\hline
\end{tabular}

TABLE 6: The code of the geometric structure in $\mathrm{mm}$ of the rough antenna and the optimized new one for ST5 satellite.

\begin{tabular}{lccccccccccccc}
\hline & $1\left(z_{0}\right)$ & $2\left(x_{1}\right)$ & $3\left(y_{1}\right)$ & $4\left(z_{1}\right)$ & $5\left(x_{2}\right)$ & $6\left(y_{2}\right)$ & $7\left(z_{2}\right)$ & $8\left(x_{3}\right)$ & $9\left(y_{3}\right)$ & $10\left(z_{3}\right)$ & $11\left(x_{4}\right)$ & $12\left(y_{4}\right)$ & $13\left(z_{4}\right)$ \\
\hline Initial & 3.00 & 1.50 & 5.37 & 20.13 & 15.02 & 2.00 & 10.04 & 8.71 & 9.20 & 3.07 & 12.47 & 6.41 & 21.40 \\
New & 4.00 & 2.00 & 5.37 & 19.63 & 14.52 & 2.00 & 9.54 & 8.21 & 9.70 & 3.57 & 12.97 & 6.41 & 20.90 \\
\hline
\end{tabular}

TABLE 7: Comparison of VSWR between the roughly evolved antenna and the orthogonally designed one.

\begin{tabular}{lcc}
\hline & Roughly evolved & Orthogonally designed \\
\hline $7209 \mathrm{MHz}$ frequency & 2.19 & 1.68 \\
$8470 \mathrm{MHz}$ frequency & 1.44 & 1.61 \\
\hline
\end{tabular}

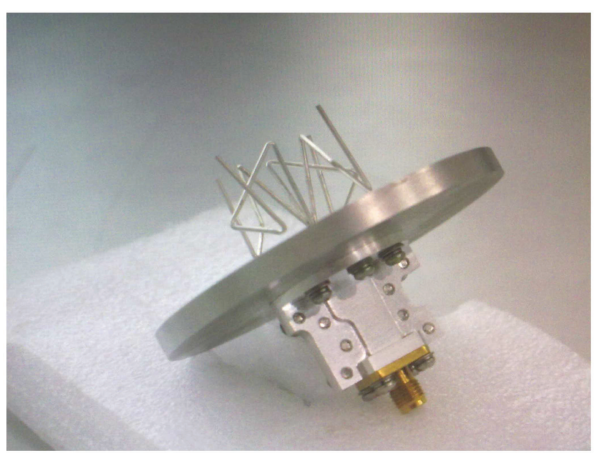

FIgURE 6: Photograph of prototype of the antenna improved by the OMD method.

The code of the roughly evolved antenna is shown in Table 6. We relax each factor of the rough antenna upper-off or lower-off $0.5 \mathrm{~mm}$ except the first feeder. Then each factor now takes the 3 levels seen in Table 5. The search space $\Omega$ is the set of all the possible combinations for all the factors at each level. The size of $\Omega$ is $\|\Omega\|=3^{13}$, a big search space.

The objective takes a summary of the penalties of the gains and VSWRs according to the specification in Table 4.

The gains are sampled in $5^{\circ}$ increments over region $40^{\circ} \leq$ $\theta \leq 80^{\circ}$ and $0^{\circ} \leq \phi \leq 360^{\circ}$. If a gain is less than $0.5 \mathrm{dBic}$, a penalty value will be given as follows:

$$
\text { penalty }_{G}(i, j)= \begin{cases}0 & \text { gain }(i, j) \geq 0.5 \\ 0.5-\operatorname{gain}(i, j) & \text { gain }(i, j)<0.5\end{cases}
$$

where gain $(i, j)$ is the gain at direction $\theta=40^{\circ}+5 i, \phi=5 j$; $i=0,1, \ldots, 8, j=0,1, \ldots, 71$.
Averaging the normalized penalties of the gains over the region, we get

$$
M P_{\text {gain }}=\frac{1}{9 * 72} \sum_{i=0}^{8} \sum_{j=0}^{71} \frac{\text { penalty }_{G}(i, j)}{\operatorname{MAX}},
$$

where MAX $=\max _{0 \leq i \leq 8,0 \leq j \leq 71}\left\{\right.$ penalty $\left._{G}(i, j)\right\}$.

Denote the average value at frequency $7209 \mathrm{MHz}$ as $M P_{\text {gain,7209 }}$ and at frequency $8740 \mathrm{MHz}$ as $M P_{\text {gain, } 8740}$.

Regarding VSWR, a penalty value is given if the VSWR is larger than 1.5 at frequency $7209 \mathrm{MHz}$ and larger than 1.2 at frequency $8740 \mathrm{MHz}$ as follows:

$$
\begin{aligned}
P_{\mathrm{VSWR}, 7209} & = \begin{cases}0 & \text { gain }(i, j) \leq 1.5 \\
\text { VSWR }-1.5 & \text { gain }(i, j)>1.5,\end{cases} \\
P_{\mathrm{VSWR}, 8740} & = \begin{cases}0 & \text { gain }(i, j) \leq 1.2 \\
\text { VSWR }-1.2 & \text { gain }(i, j)>1.2 .\end{cases}
\end{aligned}
$$

Summarizing the penalties of both gain and VSWR at frequencies both $7209 \mathrm{MHz}$ and $8740 \mathrm{MHz}$, we get objective

$$
\begin{aligned}
\min f(\vec{x})= & M P_{\text {gain }, 7209}+M P_{\text {gain }, 8740} \\
& +P_{\mathrm{VSWR}, 7209}+P_{\mathrm{VSWR}, 8740},
\end{aligned}
$$

where $\vec{x} \in \Omega$.

Note. The optimization is a minimization not maximization.

4.2. Orthogonal Experiments for ST5 Antenna Design. Given the level $Q=3$ and number of factors $N=13$, we have $J=3$ by (3). And by Algorithm 1 or (2), we have $M=$ $Q^{J}=27$. Then by using ODM with 27 experiments (that is 27 simulations of the antennas by using HFSS), a prospective combination (antenna) is found.

The code of the orthogonally designed antenna is shown in the last row in Table 6 . The antenna is pictured in the right plot of Figure 3. The gains at frequency $7209 \mathrm{MHz}$ are shown in the right subgraph of Figure 4, and the right subgraph of Figure 5 is the gains at frequency $8740 \mathrm{MHz}$. The VSWRs are shown in Table 7.

The objective $f$ (see (9)) is shown in Table 8. It shows that the orthogonally designed antenna is better than the roughly evolved one. 
4.3. Discussion. Some comments on ODM in optimizing antenna design are given in the following.

(1) Determining factors (variables) $N$, levels of each factor $Q$, and objective: This is the preparation step. The size of the search space is exponent of the number of factors with the number of levels as base $\|\Omega\|=Q^{N}$. The number of factors and the number of levels of each factor could not be too big. The search space of the ST5 application is $\|\Omega\|=3^{13}$ where $N=13, Q=3$.

(2) Determining the size of orthogonal array $Q^{J}: Q$ is given according to the above item. $J$ is chosen as smaller as possible to get the smallest orthogonal array since a simulation is time consuming. $Q=3, J=$ 3 in the ST5 application. Then $3^{3}=27$ simulations are done in the orthogonal experiments. It is far less than the search space $3^{3} \ll 3^{13}$. However, the objective value of the orthogonally designed antenna is better than that of the roughly evolved one.

(3) Finding a prospective combination (antenna structure) by statistical summary from very small samples: In the case of linear or quadratic objective, the statistical summary is proven right. It is not proven right in other cases. But a derivable objective can be approached by a quadratic over a small neighbor region. That is, the statistical summary in this paper is reliable. Anyway, we must do experiment to verify the result finding from the summary. Fortunately, the orthogonally designed antenna is better than the roughly evolved one in the ST5 application problem. Figure 6 is the prototype of the orthogonally designed antenna.

\section{Conclusion}

The idea of the orthogonal design method in designing antenna is mainly as follows.

(1) The geometrical structure of the antenna is parameterized into factors and each factor is quantized into discrete levels; the requirements specified for the antenna are functionalized as objective. The number of factors and number of levels should not be too big because of the exponent increase of the search space with the number of factors where the base is the number of levels.

(2) Since an electromagnetic simulation lasts usually for minutes or even hours, smallest orthogonal array is taken. This paper offered an algorithm to create a class of orthogonal arrays and a way to determine the smallest orthogonal array for the ODM finishing in endurable time.

(3) By using the ODM, a potential good antenna could be found with very small number of electromagnetics simulations over a very large design space.

(4) The objective value of the orthogonally designed antenna is better than that of the rough one, and then
TABLE 8: Comparison of objective values between the roughly evolved antenna and the orthogonally designed one.

\begin{tabular}{lccccc}
\hline & $M P_{\text {gain,7209 }}$ & $M P_{\text {gain,8740 }}$ & $P_{\text {VSWR,7209 }}$ & $P_{\text {VSWR }, 8740}$ & $f$ \\
\hline $\begin{array}{l}\text { Roughly } \\
\text { evolved }\end{array}$ & 0.0 & 0.0 & 0.69 & 0.24 & 0.93 \\
$\begin{array}{l}\text { Orthogonally } \\
\text { designed }\end{array}$ & 0.0 & 0.0 & 0.18 & 0.41 & 0.59 \\
\hline
\end{tabular}

the prototype was made by the orthogonally designed antenna.

Future work is explained as follows.

(1) Antenna design is an expensive problem; see literature $[28,29]$. ODM will be implemented in parallel for antenna design, and surrogate-assisted model is another way to reduce running time.

(2) Another future work is to compromise conflict between gains, VSWR, axial ratio, and so on in determining objective.

\section{Conflict of Interests}

The authors declare that there is no conflict of interests regarding the publication of this paper.

\section{Acknowledgment}

This work was supported by the National Natural Science Foundation of China (nos. 61271140, 61203306, and 60871021).

\section{References}

[1] A. S. Hedayat, N. J. A. Sloane, and J. Stufken, Orthogonal Arrays: Theory and Applications, Springer, New York, NY, USA, 1999.

[2] G. Taguchi, Introduction to Quality Engineering: Designing Quality into Products and Process, Asian Productivity Organization, Tokyo, Japan, 1986.

[3] L.-W. Chung, K.-L. Lin, T. C.-C. Yang, and M.-R. Lee, "Orthogonal array optimization of microwave-assisted derivatization for determination of trace amphetamine and methamphetamine using negative chemical ionization gas chromatography-mass spectrometry," Journal of Chromatography A, vol. 1216, no. 18, pp. 4083-4089, 2009.

[4] J. Y. X. Zhan, K. Y. Z. Zheng, K. Y. Zhu et al., "Chemical and biological assessment of angelicae sinensis radix after processing with wine: an orthogonal array design to reveal the optimized conditions," Journal of Agricultural and Food Chemistry, vol. 59, no. 11, pp. 6091-6098, 2011.

[5] F. Lucas and J. Xiaoyi, "Orthogonal design of experiments for parameter learning in image segmentation," Signal Processing, vol. 93, no. 6, pp. 1694-1704, 2013.

[6] W. Guo, M. Hua, P. W.-T. Tse, and A. C. K. Mok, "Process parameters selection for laser polishing DF2 (AISI O1) by Nd:YAG pulsed laser using orthogonal design," International Journal of Advanced Manufacturing Technology, vol. 59, no. 912, pp. 10091023, 2012. 
[7] L. Lazic and N. Mastorakis, "Orthogonal Array application for optimal combination of software defect detection techniques choices," WSEAS Transactions on Computers, vol. 7, no. 8, pp. 1319-1336, 2008.

[8] Q. Zhang and Y.-W. Leung, "An orthogonal genetic algorithm for multimedia multicast routing," IEEE Transactions on Evolutionary Computation, vol. 3, no. 1, pp. 53-62, 1999.

[9] G. DePinto and J. Wilson, "Optimization of LPCVD silicon nitride deposition process by use of designed experiments," in Proceedings of Advanced Semiconductor Manufacturing Conference and Workshop, pp. 47-53, 1990.

[10] Z. Tong, M. N. Akram, and X. Chen, "Speckle reduction using orthogonal arrays in laser projectors," Applied Optics, vol. 49, no. 33, pp. 6425-6429, 2010.

[11] Y. Zhu, P. Zeng, and K. Jennings, "Optimal compound orthogonal arrays and single arrays for robust parameter design experiments," Technometrics, vol. 49, no. 4, pp. 440-453, 2007.

[12] E. D. Schoen, P. T. Eendebak, and M. V. M. Nguyen, "Complete enumeration of pure-level and mixed-level orthogonal arrays," Journal of Combinatorial Designs, vol. 18, no. 2, pp. 123-140, 2010.

[13] E. Agoston and S. James, Introduction to Evolutionary Computing, Springer, 2003.

[14] D. S. Linden and E. E. Altshuler, "Automating wire antenna design using genetic algorithms," Microwave Journal, vol. 39, no. 3, pp. 74-86, 1996.

[15] J. D. Lohn, W. F. Kraus, and D. S. Linden, "Evolutionary optimization of a quadrifilar helical antenna," in Proceedings of the IEEE Antennas and Propagation Society International Symposium, pp. 814-817, June 2002.

[16] S. Zeng, Z. Liu, C. Li, Q. Zhang, and W. Wang, "An evolutionary algorithm and its application in antenna design," Journal of Bioinformatics and Intelligent Control, vol. 1, no. 2, pp. 129-137, 2012.

[17] J. Kennedy and R. C. Eberhart, Swarm Intelligence, Morgan Kaufmann, 2001.

[18] Z. Cui and X. Cai, "Integral particle swarm optimization with dispersed accelerator information," Fundamenta Informaticae, vol. 95, no. 4, pp. 427-447, 2009.

[19] E. Garcia-Gonzalo and J. L. Fernandez-Martinez, "A brief historical review of particle swarm optimization (PSO)," Journal of Bioinformatics and Intelligent Control, vol. 1, no. 1, pp. 3-16, 2012.

[20] T. S. J. Laseetha and R. Sukanesh, "Investigations on the synthesis of uniform linear antenna array using biogeographybased ptimization techniques," International Journal of Bio-Inspired Computation, vol. 4, no. 2, pp. 119-130, 2012.

[21] D. Mandal, S. P. Ghoshal, and A. K. Bhattacharjee, "Optimized radii and excitations with concentric circular antenna array for maximum sidelobe level reduction using wavelet mutation based particle swarm optimization techniques," Telecommunication Systems, vol. 52, no. 4, pp. 2015-2025, 2013.

[22] R. Storn and K. Price, "Differential evolution-a simple and efficient heuristic for global optimization over continuous spaces," Journal of Global Optimization, vol. 11, no. 4, pp. 341-359, 1997.

[23] Y. W. Zhong, L. J. Wang, C. Y. Wang, and H. Zhang, "Multi-agent simulated annealing algorithm based on differential evolution algorithm," International Journal of Bio-Inspired Computation, vol. 4, no. 4, pp. 217-228, 2012.

[24] L. Shu-Han, C. Chien-Ching, and H. Min-Hui, "Comparison of dynamic differential evolution and genetic algorithm for
MIMO-WLAN transmitter antenna location in indoor environment," Wireless Personal Communications, vol. 71, no. 4, pp. 2677-2691, 2013.

[25] D. C. Montgomery, Design and Analysis of Experiments, Wiley, New York, NY, USA, 3rd edition, 1991.

[26] http://www.nasa.gov/mission.

[27] G. S. Hornby, J. D. Lohn, and D. S. Linden, "Computer-Automated evolution of an X-band antenna for NASA's space technology 5mission," Evolutionary Computation, vol. 19, no. 1, pp. $1-23,2011$.

[28] Y. Jin, "Surrogate-assisted evolutionary computation: recent advances and future challenges," Swarm and Evolutionary Computation, vol. 1, no. 2, pp. 61-70, 2011.

[29] J. He and X. Yao, "Analysis of scalable parallel evolutionary algorithms," in Proceedings of the IEEE Congress on Evolutionary Computation (CEC '06), pp. 120-127, July 2006. 

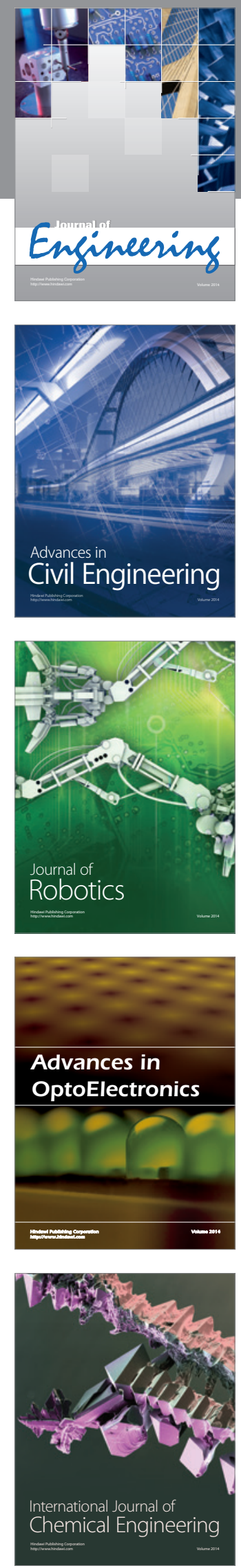

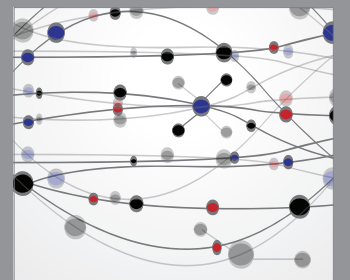

The Scientific World Journal
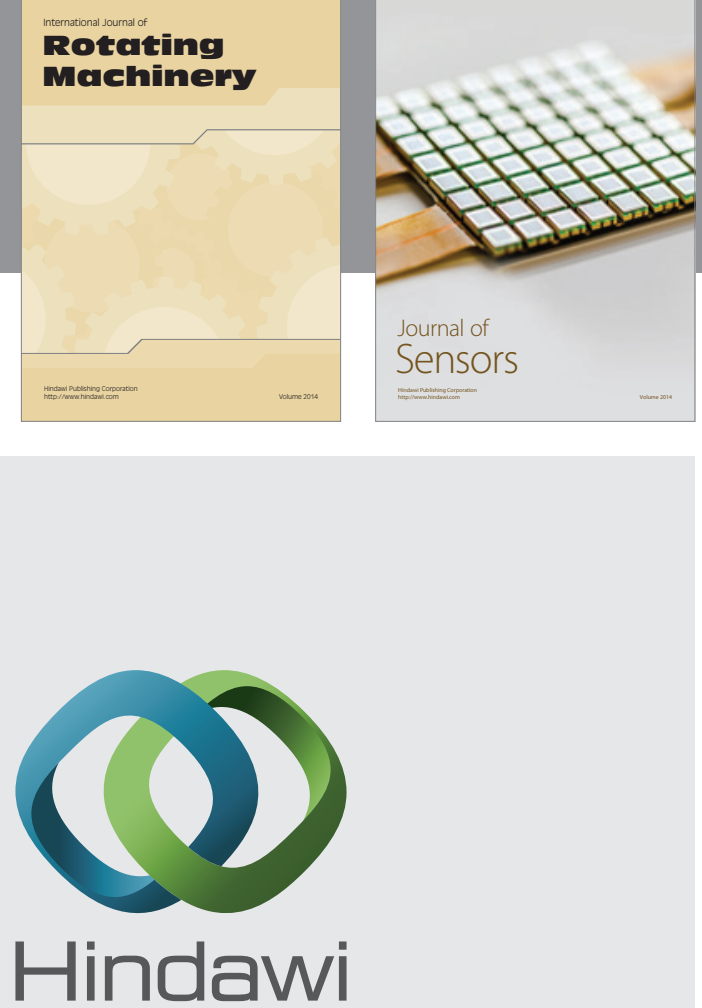

Submit your manuscripts at http://www.hindawi.com
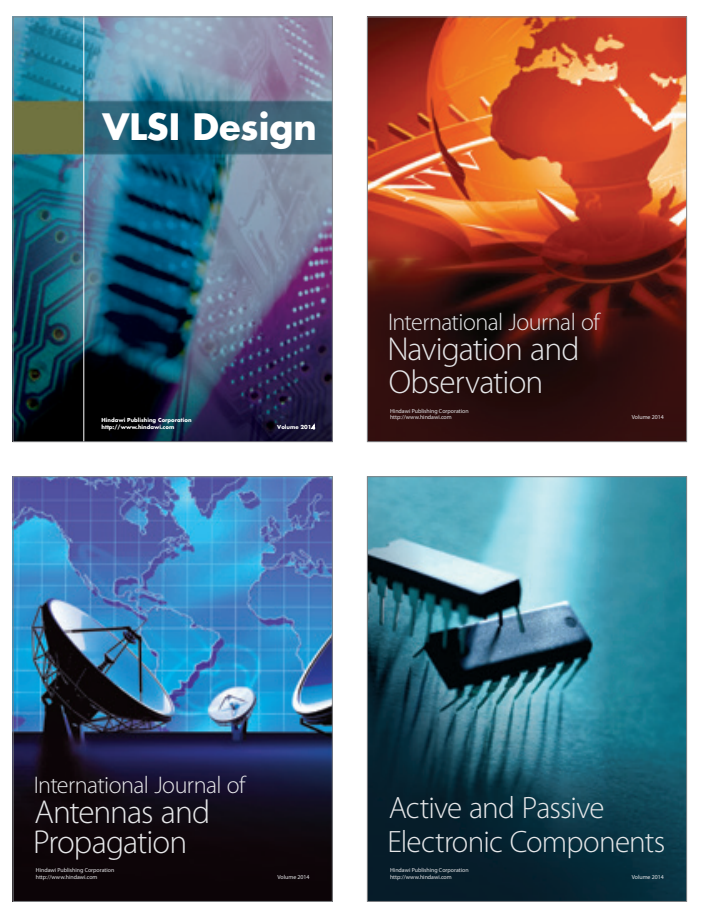
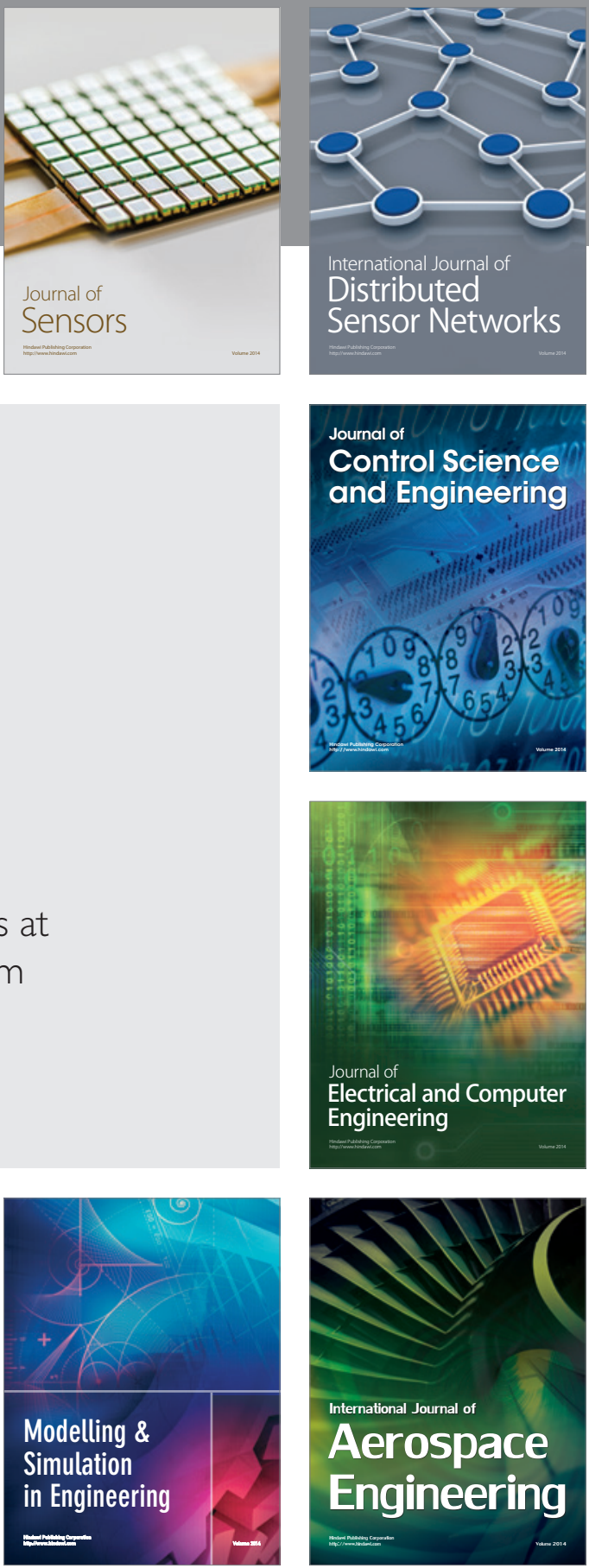

Journal of

Control Science

and Engineering
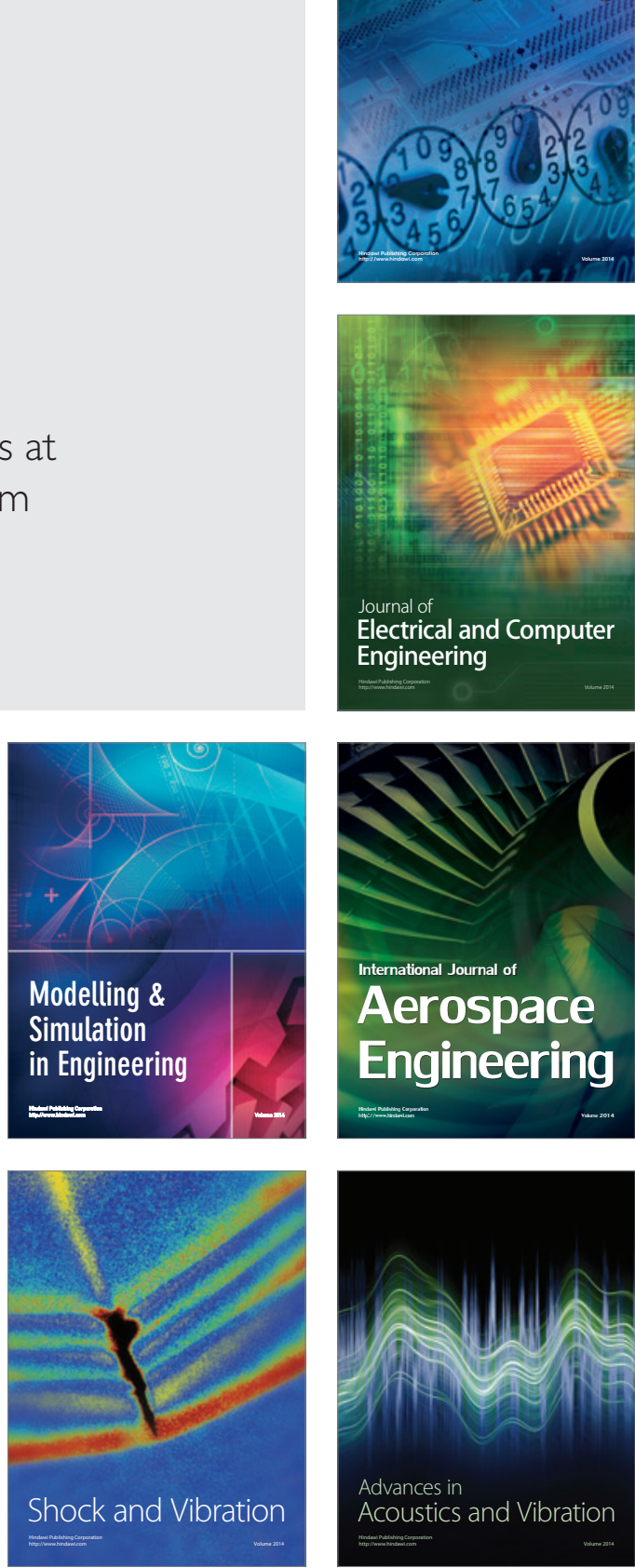